\title{
Dipole formation at organic/metal interfaces with pre-deposited and post-deposited metal
}

\author{
Zhanhao Hu, Zhiming Zhong, Kai Zhang, Zhicheng Hu, Chen Song, Fei Huang, Junbiao Peng, Jian Wang \\ and Yong Cao
}

In organic electronic devices, the interfacial dipole at organic/metal interfaces is critical in determining the carrier injection or extraction that limits the performance of the device. A novel technique to enable the direct measurement of underburied dipoles is developed and demonstrated. By tilting the shadow mask by a small angle, metal atoms diffuse into the opening slit to form an ultrathin metal layer during the evaporation process. As the ultrathin metal layer cannot screen out the dipole-induced surface work function change, the dipole strength and direction at the organic/metal interface can be revealed. It was found that the polarity of the organic material, the Fermi-level pinning and the interface morphology all play important roles in dipole formation. By comparing the energy level shifts at the organic/pre-deposited metal and organic/post-deposited metal interfaces, the dipole formed by molecular interactions could be distinguished from the dipole formed by Fermi-level pinning.

NPG Asia Materials (2017) 9, e379; doi:10.1038/am.2017.56; published online 19 May 2017

\section{INTRODUCTION}

In organic electronic devices, such as organic solar cells and organic light-emitting diodes, energy-level alignment at the organic/metal interface is critical for device performance because it determines the carrier injection barrier and device built-in voltage. ${ }^{1,2}$ A small energylevel offset between the organic material's charge transport level and the metal's Fermi level is always favored to form an ohmic contact at the interface. Therefore, metals with low work function (WF), such as $\mathrm{Ba}, \mathrm{Ca}, \mathrm{Li}, \mathrm{Cs}$ and $\mathrm{Al}$, are popular choices for cathodes to facilitate electron injection or extraction. ${ }^{3-5}$ However, low WF metals are intrinsically unstable. As a consequence, much effort has been made to develop electrode modification materials to enable the use of stable but high WF metals as cathodes. ${ }^{6}$ Both inorganic materials, such as alkali-metal salts and metal oxides, and organic materials, such as organic surfactants, have been successfully applied in devices. ${ }^{7-12}$ Through the formation of a dipole layer at the organic/metal interface, induced by the interfacial interaction, the interfacial energy-level alignment is modified, leading to a reduction in the charge injection barrier. ${ }^{1,13}$ Various molecular structures are being examined to find the best modification materials for different devices.

To select the desired interfacial material, the most common characterization is measuring the WF change on substrates of the cathode metal before and after depositing the interfacial materials on top $^{14,15}$ (Figure 1a). A reduced WF indicates that the electron injection barrier is lowered and hence can be used at the cathode. In contrast, an increased WF implies that the material can be used to enhance hole injection at the anode. The deduction is straightforward for inverted devices for which the layer deposition sequence, that is, cathode followed by the interfacial material, is the same as that in the characterization process (Figure 1). However, for regular devices, whereby the interfacial layer is deposited before the cathode, the deduction may not reveal the actual energy alignment across the organic/metal interface. ${ }^{1}$ Various chemical or physical interactions can occur at the interface of the post-deposited electrode that alter the energy-level alignment. Damage to the conjugated bonding structure from hot metal atoms, metal diffusion, metal-induced gap states or different metal morphologies on organic materials has been found to influence the interfacial energetics. ${ }^{16-19}$ Thus, the metal deposition sequence may lead to different charge injection barriers.

Although most studies have examined the organic/metal interface with a pre-deposited electrode, relatively few attempts have been made to examine the interface with post-deposited metals. The difficulty with conducting such studies originates from the spontaneous Fermi-level alignment between the metal and the conductive substrate (Figure 1b). As a consequence, the formed dipole at the organic/metal interface will be screened out and cannot be detected by measuring the WF change on top of the metal layer. One solution to observe the embedded dipole is by peeling off the post-deposited metal together with the interfacial material to expose the organic/metal interface. ${ }^{20,21}$ The modified metal WF can then be directly obtained. However, to separate the films at the desired interfaces, careful control of the adhesion between film layers is necessary that may not be possible for many materials. An alternative approach is to directly measure the dipole before it is screened out by the metal. In that approach, the WF change is monitored during metal deposition. ${ }^{17,22-24}$ The overall process requires precise film thickness control to deposit 

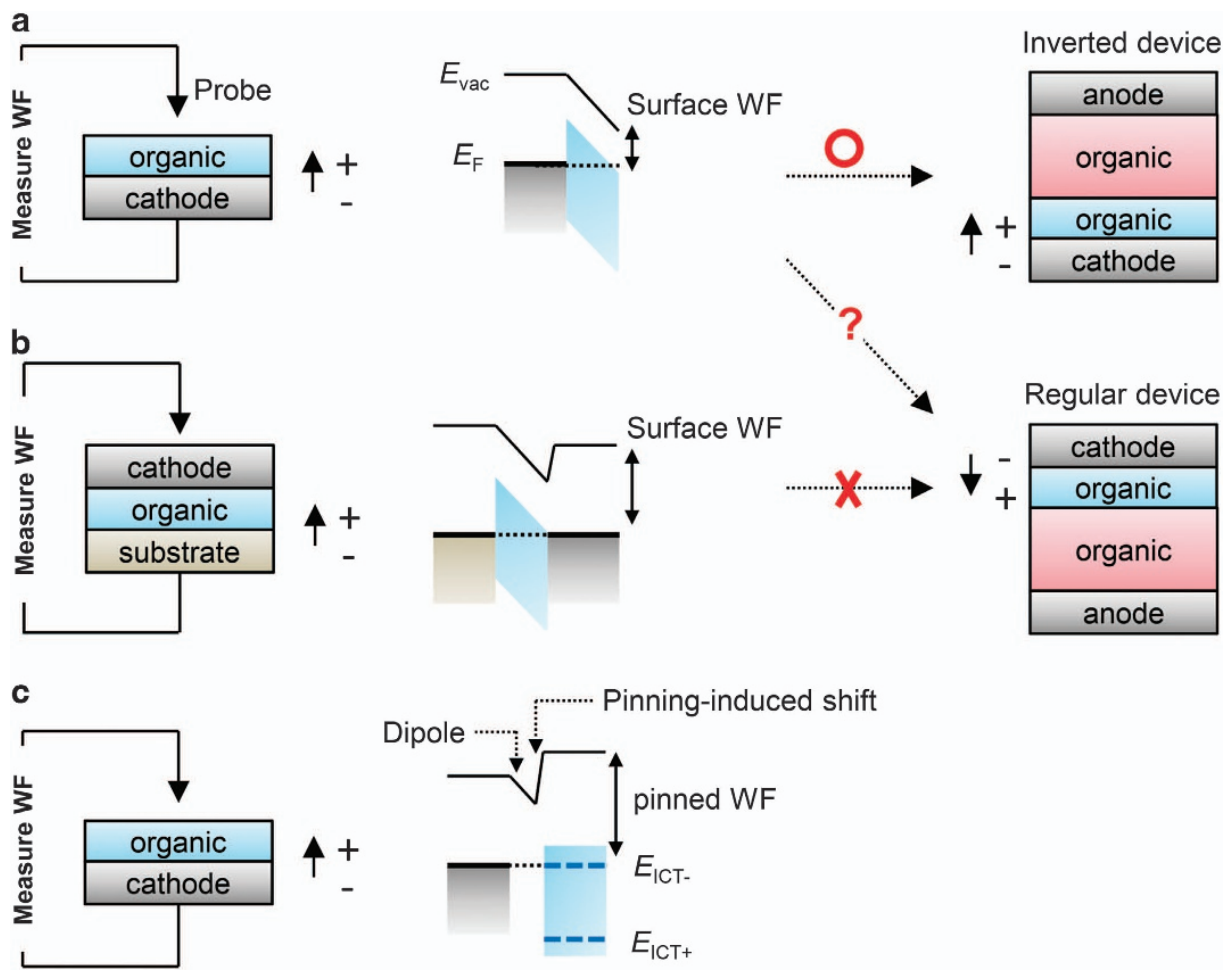

Figure 1 (a) The conventional technique used to probe organic/metal dipoles is to measure the surface work function (WF) change of the cathode metal modified by organic interfacial materials. The layer deposition sequence is the same as that in the inverted device structure; hence, the energy diagram at the interface can be applied to the device. (b) In contrast, in regular device structures, the cathode metal is deposited after the organic layer. Thus, the measurement result may not be directly applied to the device interpretation. Because of the Fermi-level alignment between the cathode metal and conductive substrate of the sample, the embedded dipole at the post-deposited metal interface cannot be detected. (c) When the metal's Fermi level is pinned to the integer charge transfer level of the organic material, the measured WF is pinned, and the dipole is screened out.

the metal monolayer by monolayer. Only a few organic materials have been examined using this method because of the rigorous requirements of the experimental set-up.

In our contribution, we propose a novel scheme to avoid the thick metal screening effect and to measure the dipole directly on the organic/post-deposited metal interface. To deposit the metal on top of the organic material, a tilted shadow mask is employed. During the metal deposition process, metal vapor diffuses into the shadow area to form an ultrathin metal layer. As the dipole formation at the organic/ metal interface has yet to be screened out by the ultrathin metal layer, the dipole can be directly measured in the form of the surface potential change using a Kelvin probe. ${ }^{23}$ Typical organic materials, with properties such as conjugated and nonconjugated, polar and nonpolar and semiconducting and insulating, are studied, whereas $\mathrm{Al}$ is selected as the cathode metal because it is the most commonly used cathode metal in organic devices. It is found that for nonpolar materials, there is a negligible potential shift at the organic/Al interfaces. For polar materials, the orientation and strength of the dipoles measured are similar at both the organic/pre-deposited $\mathrm{Al}$ interface and the organic/post-deposited $\mathrm{Al}$ interface.

Furthermore, many low-bandgap materials have been developed in recent years to enhance light harvesting in solar cells. ${ }^{25}$ Low-bandgap materials often possess a low negative integer charge transfer level ( $\left.\mathrm{E}_{\mathrm{ICT}-}\right)$ and/or a high positive integer charge transfer level $\left(\mathrm{E}_{\mathrm{ICT}+}\right)$. As a consequence, Fermi-level pinning is easily formed at the organic/metal interface if the metal Fermi level is above $\mathrm{E}_{\mathrm{ICT}-}$ or below $\mathrm{E}_{\mathrm{ICT}+}$. As illustrated in Figure 1c, upon Fermi-level pinning, the interfacial dipole is screened out by the pinning-induced potential shift. Therefore, it cannot be detected by measuring the WF change on the organic/pre-deposited metal surface. ${ }^{26}$ In contrast, our developed method can elucidate dipole formation at the interface and hence reveal the interaction between the organic molecules and metal atoms at the organic/metal interface.

\section{MATERIALS AND METHODS}

\section{Materials and sample preparation}

The organic materials, poly[2-methoxy-5-(2'-ethylhexyloxy)-p-phenylene vinylene] (MEH-PPV) (0.5 wt $\%$ in chlorobenzene), poly[2-(4-(30,70-dimethyloctyloxy)-phenyl)-p-phenylenevinylene) (P-PPV) (0.8\% in p-xylene), poly [[4,8-bis[(2-ethylhexyl)oxy]benzo[1,2-b:4,5-b'] dithiophene-2,6-diyl] [3-fluoro2-[(2-ethylhexyl)carbonyl] thieno[3,4-b]thiophenediyl]] (PTB7) (1 wt $\%$ in

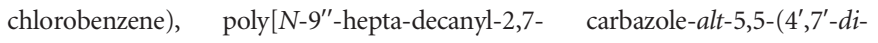
2-thienyl-2', $1^{\prime}, 3^{\prime}$-benzothiadiazole) (PCDTBT) (0.2 wt $\%$ in o-dichlorobenzene) and [6,6]-phenyl C71-butyric acid methyl ester ( $\left.\mathrm{PC}_{71} \mathrm{BM}\right) \quad(0.1 \quad \mathrm{wt} \%$ in chloroform), were purchased from 1-Material (Dorval, Quebec, Canada). Benzoic acids with $-\mathrm{OCH}_{3}$ and $-\mathrm{CN}$ end groups $\left(\mathrm{BA}-\mathrm{OCH}_{3}\right.$ and $\left.\mathrm{BA}-\mathrm{CN}\right)$ were purchased from J\&K Scientific (Beijing, China). The interfacial materials, poly[(9,9-bis(3'-(N,N-dimethylamino)propyl)-2,7-fluorene)-alt-2,7(9,9-dioctylfluorene)] (PFN) (0.2 wt\% in methanol), poly[(9,9-bis(3'-((N,Ndimethyl)- $N$-ethylammonium)-propyl)-2,7-fluorene)-alt-2,7-(9,9-dioctylfluorene)] (PFNBr) (0.05 wt\% in water) and poly[9,9-bis(4'-butanoatel)fluoreneco-alt-2,7-(9,9-dioctylfluorene)] sodium salt (PFCOONa) (0.05 wt $\%$ in water), were synthesized in our laboratory, whereas polyetherimide (PEI) $(0.01 \%$ in methanol) was purchased from Sigma-Aldrich (St Louis, MO, USA). The insulating polymers, poly(methyl methacrylate) (PMMA) (5 wt $\%$ in $n$-butylacetate), poly(ethylene oxide) (PEO) (0.5 wt $\%$ in methanol) and polyvinylpyrrolidone (PVP) (0.5 wt $\%$ in ethanol), were purchased from Sigma-Aldrich. 
a

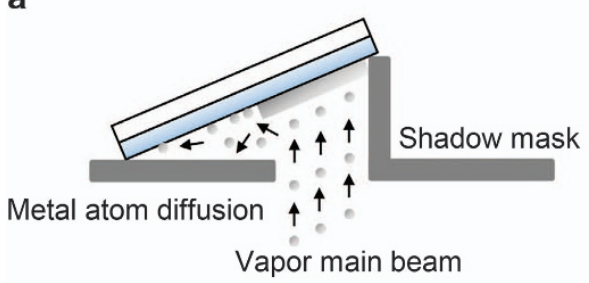

b

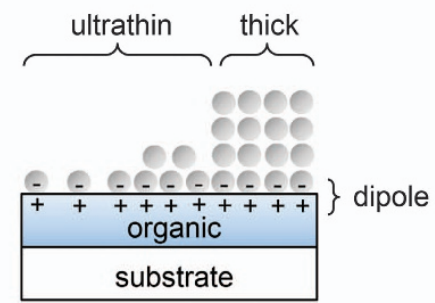

C

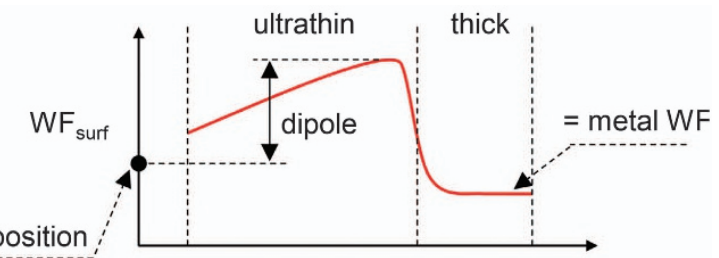

(1)
(2) Location

(3) d

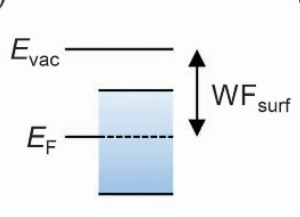

(2)

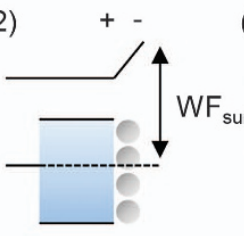

(3) +
$-$

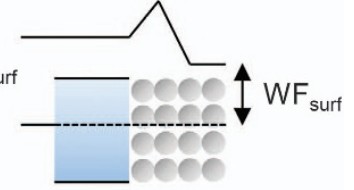

Figure 2 (a) Schematic of the metal deposition method. The substrate is propped up at one side to allow a small amount of metal atoms to diffuse into the shadowed areas. (b) The area directly above the mask opening is deposited with a thick metal layer. In the shadowed area, an ultrathin metal layer of atom thickness is adsorbed. The metal atom coverage increases from the far end to the end near the slit opening. A dipole is formed that is directed from the metal to the organic layer, as illustrated. An opposite dipole direction is also possible for different organic materials. (c) The surface work function (WF) is scanned from side to side across the sample surface. In the ultrathin metal region, the surface WF equals the substrate WF plus the dipole. In the thick metal region, the surface WF returns to the intrinsic metal WF as a consequence of Fermi-level alignment. (d) The energy diagrams for each region on the surface: (1) WF on the organic surface before metal deposition, (2) in the ultrathin metal region and (3) in the thick metal region.

To fabricate the organic/post-deposited metal samples, the organic materials are spin-coated from solution onto precleaned indium tin oxide (ITO) substrates before metal deposition. The self-assembly molecules $\mathrm{BA}-\mathrm{OCH}_{3}$ and BA-CN are first dissolved in ethanol in a $1 \mathrm{~mm}$ concentration. Then, the solutions are spin-coated onto the plasma-treated ITO substrates and rinsed in ethanol solvent to obtain monolayer adsorption. To fabricate the organic/pre-deposited metal samples, $\mathrm{Al}$ is thermally deposited onto precleaned ITO substrates in a vacuum chamber before spin-coating the organic materials on top.

\section{Characterization}

The surface WF is measured using a Kelvin Probe (KP5050 from KP Technology, Wick, UK) with a probe tip diameter of $2 \mathrm{~mm}$, calibrated by highly oriented pyrolytic graphite $(4.6 \mathrm{eV})$. The electric potential change at the organic/post-deposited metal interface is derived from the difference in the surface WF before and after ultrathin metal deposition. A minimum of three samples are measured for each configuration to guarantee the reliability of the results.

The existence of $\mathrm{Al}$ at the shadowed area of the sample is detected by X-ray photoelectron spectroscopy (XPS) (ESCALAB 250Xi, Thermo-Fisher Scientific, Waltham, MA, USA). The sample for cross-sectional transmission electron microscopy imaging (Titan Themis 200, FEI, Hillsboro, OR, USA) has a structure of ITO/poly(9-vinylcarbazole)/Al capped with chromium and carbon protective layers. $\mathrm{Al}$ is deposited following the method described in the next section of the paper. Ion milling is carried out at locations in the shadowed area.

Atomic force microscopy images are obtained using a Dimension Icon microscope (Bruker Corporation, Billerica, MA, USA) in tapping mode.

\section{RESULTS AND DISCUSSION}

Our novel method is schematically illustrated in Figure 2a. First, the organic material is coated on a conductive substrate, such as ITO. The substrate is then placed on a shadow mask and propped up on one side. The tilt angle is $\sim 9^{\circ}$, and a narrow slit is left between the substrate and the mask. The substrate and mask are subsequently transferred into the vacuum chamber and pumped down to $\sim 10^{-4} \mathrm{~Pa}$. Al is thermally evaporated at a rate of $0.3 \pm 0.1 \mathrm{~nm} \mathrm{~s}^{-1}$ until a total thickness of $\sim 120 \mathrm{~nm}$ is reached. Notably, a thicker metal layer does not affect the results. During evaporation, owing to the large mean free path of $\mathrm{Al}$ atoms $(\sim 10 \mathrm{~m}),{ }^{27}$ most atoms are deposited on the substrate directly above the mask opening, whereas a very small amount of metal vapor diffuse through the narrow slit, cool, and condense onto the shadow area. Al adsorption is evidenced by X-ray photoelectron spectroscopy. As shown in Figure 3, in the thick $\mathrm{Al}$ region, $\mathrm{Al} 2 \mathrm{p}$ exhibits two peaks at 71.7 and $74.3 \mathrm{eV}$ that correspond to metallic $\mathrm{Al}$ and oxidized $\mathrm{Al}$, respectively. In the shadowed region, only the oxidized component is observed, indicating that the adsorbed layer is ultrathin. The $\mathrm{Al} 2 \mathrm{p}$ peak intensity at the far side from the thick $\mathrm{Al}$ (region 1) is lower than that at the near side (region 2), confirming that less $\mathrm{Al}$ is adsorbed in the region far away from the slit opening.

To examine the morphology of the adsorbed metal, cross-sectional transmission electron microscopy images are obtained. However, because of the ultrathin nature of the adsorbed $\mathrm{Al}$ species and the low contrast between oxidized $\mathrm{Al}$ and the organic layer, the adsorbed layer cannot be resolved (Supplementary Figure S1). By using atomic force microscopy, the adsorbed species can be observed in the height and phase images, as shown in Figure 4. To better detect the ultrathin $\mathrm{Al}$, surface oxidized silicon $\left(\mathrm{Si} / \mathrm{SiO}_{2}\right)$ is used as the substrate owing to its superior surface smoothness. Before metal deposition, the bare substrate shows a root mean square roughness $\left(\mathrm{R}_{\mathrm{RMS}}\right)$ of $0.198 \mathrm{~nm}$. After metal deposition, the shadowed area shows grainy features, and the $\mathrm{R}_{\mathrm{RMS}}$ slightly increases to $0.434 \mathrm{~nm}$, confirming the adsorption of the metal. The thickness is estimated to be below $1 \mathrm{~nm}$. Atomic force microscopy images of the Al-adsorbed ITO and organic substrates are also examined and show similar morphologies (Supplementary Figures S2-S4).

The surface WF measurement, schematically illustrated in Figures $2 b$ and $c$, can uncover the increasing coverage of the metal atoms on the organic layer from the far end to the end near the slit opening. As the ultrathin $\mathrm{Al}$ layer is of atomic thickness and is spontaneously oxidized in the chamber, the metallic properties of $\mathrm{Al}$ are not present, as indicated by the XPS spectrum in Figure 3 and an ultraviolet photoelectron spectroscopy study. ${ }^{23}$ Without the metal 


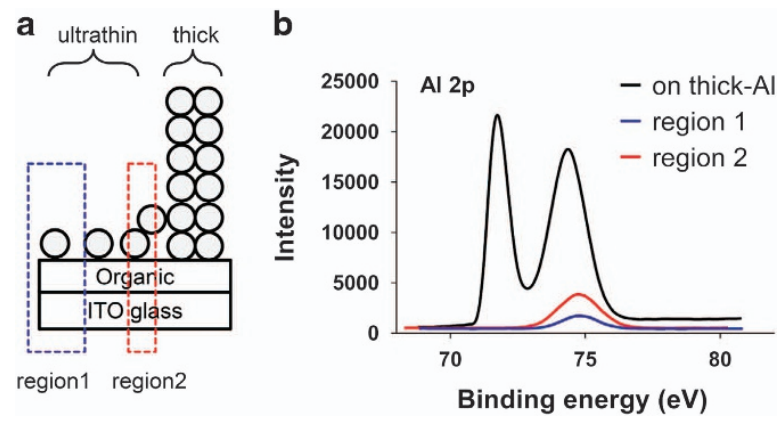

Figure 3 (a) Schematic of the deposited Al film on the indium tin oxide (ITO)/organic substrate. (b) X-ray photoelectron spectroscopy (XPS) spectra of ITO/MEH-PPV/PFN/(AI). Al 2p peaks are detected on the thick $\mathrm{Al}$ layer and on region 1 and region 2 on the ultrathin $\mathrm{Al}$ layer. The existence of $\mathrm{Al}$ species in the ultrathin Al layer indicates that the metal vapor can diffuse into the slit opening between the shadow mask and substrate to be adsorbed on the organic surface.

screening effect, the dipole information at the organic/metal interface can be obtained directly by comparing the surface WF before and after metal deposition. The dipole direction and strength is represented by the WF difference between the bare organic substrate and the peak (or valley if the dipole direction is opposite to that in Figure $2 \mathrm{~b}$ ) of the scanned WF on the organic/post-deposited Al sample (Figure 2c). At the boundary of the thick $\mathrm{Al}$ layer, the metallic properties of $\mathrm{Al}$ are present and screen out any electric potential change under the layer. As the result, the WF will drop steeply until it reaches the intrinsic $\mathrm{Al}$ WF. The energy diagrams at the organic interfaces without the metal layer, with an ultrathin metal layer and with a thick metal layer are depicted in Figure 2d. In the experiment, the WF is obtained from the surface potential measurement using a Kelvin probe. The maximum experimental error is $<0.1 \mathrm{eV}$. Details on the reproducibility of the data are provided in Supplementary Figures S5-S8.

As shown in Figure 5, several groups of organic materials are studied. The first row includes self-assembled molecules with well-defined polar functionalities. The self-assembled molecules have simple structures, and their molecular orientation can be controlled through the anchoring groups. Benzoic acids (BA-X) with polar end groups of electron-rich $-\mathrm{OCH}_{3}$ and electron-deficient -CN are selected. The common - $\mathrm{COOH}$ end groups of these two molecules are adsorbed on the hydroxyl-covered ITO surface through hydrogen bonds, leaving the polar ends pointed toward the free surface. ${ }^{28}$ After $\mathrm{Al}$ deposition, the WF on ITO/BA- $\mathrm{OCH}_{3}$ increases as the $\mathrm{Al}$ coverage changes from thin to thick. The black dot in the plot is the surface WF of the bare organic substrate before Al deposition. The WF difference between the bare organic substrate and the peak value of the metal-covered organic sample gives the total dipole at the organic/ metal interface. At the $\mathrm{BA}-\mathrm{OCH}_{3} / \mathrm{Al}$ interface, negative charges transfer from $-\mathrm{OCH}_{3}$ to $\mathrm{Al}$ atoms, presenting a WF change of $0.13 \mathrm{eV}$. At BA-CN/Al, an oppositely orientated dipole $(-0.21 \mathrm{eV})$ is observed, indicating negative charge transfer from $\mathrm{Al}$ to $-\mathrm{CN}$. The charge transfer is further confirmed by the XPS spectrum of the BA-CN/Al interface (Supplementary Figure S10). The results are consistent with other studies that found a reduction in the electron injection barrier using $\mathrm{BA}-\mathrm{OCH}_{3}$ as the interfacial layer and an increase in the barrier using BA-CN in organic solar cells and tunneling diode devices. ${ }^{29,30}$

Other commonly used interfacial materials also contain similar polar side chains to achieve the modification effect on the metal WF. PFN and PEI are the most popular polymeric and small-molecule
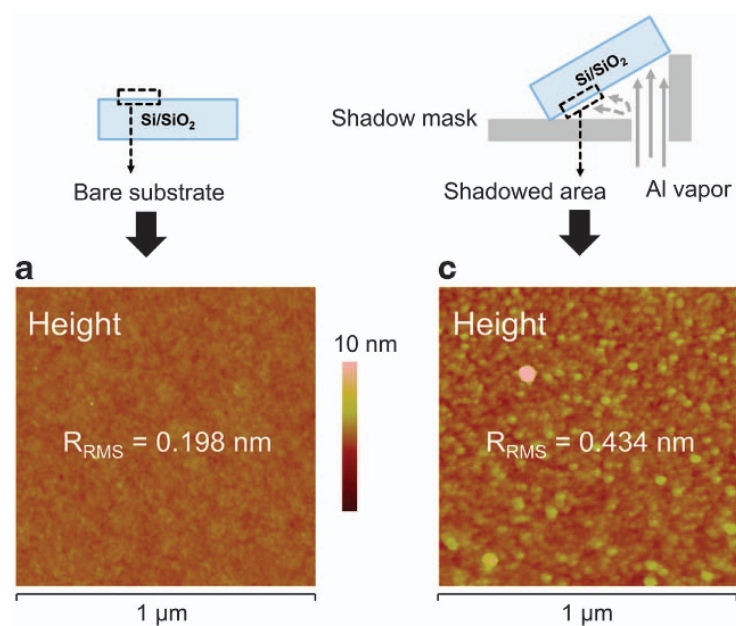

c
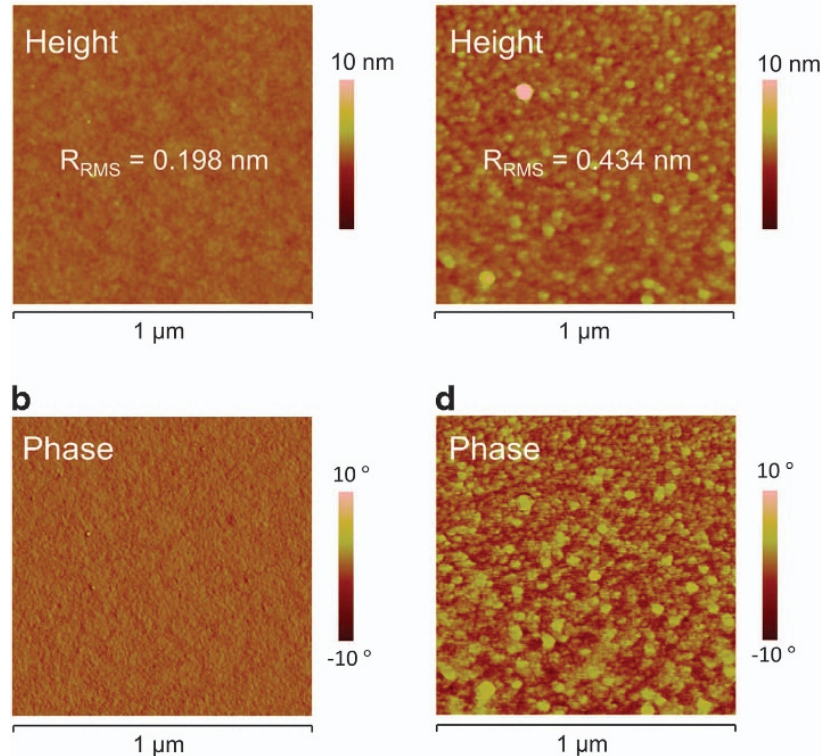

Figure 4 (a, b) Atomic force microscopy (AFM) height and phase images $(1 \mu \mathrm{m} \times 1 \mu \mathrm{m})$ on a bare $\mathrm{Si} / \mathrm{SiO}_{2}$ substrate. The surface root mean square (RMS) roughness is $0.198 \mathrm{~nm}$. (c, d) AFM height and phase images $(1 \mu \mathrm{m} \times 1 \mu \mathrm{m})$ on the shadowed area after depositing $\mathrm{Al}$ on $\mathrm{Si} / \mathrm{SiO}_{2}$. The grainy features indicate the adsorbed Al species. The surface RMS roughness is $0.434 \mathrm{~nm}$. The $\mathrm{Si} / \mathrm{SiO}_{2}$ substrate was chosen for its very smooth surface to better distinguish the adsorbed metal.

interfacial materials in organic electronic devices. ${ }^{7} 8$ It is found that the amino groups can push electrons toward the metal through Lewis acid-base interactions, forming a dipole layer that effectively reduces the cathode WF. ${ }^{8,31}$ To study dipole formation in the device structure, interfacial materials are spin-coated on a typical active layer material, MEH-PPV, followed by $\mathrm{Al}$ deposition. By scanning across PFN and PEI layers covered with an ultrathin Al layer, significant changes in the surface WF by $\sim 0.40$ and $0.45 \mathrm{eV}$ are detected (Figure 5), suggesting that the dipole is directed from the metal to the organic layer. The XPS spectrum of MEH-PPV/PFN/Al is examined in Supplementary Figure S9. The results are consistent with our earlier study using the peeling-off method to induce a reduction in the electron injection barrier of $0.4 \mathrm{eV}$ after the insertion of a PFN layer. ${ }^{21}$ The measured dipole direction at the $\mathrm{PEI} / \mathrm{Al}$ interface is also consistent with that in other studies in which a reduced electron injection barrier was found. ${ }^{8}$

The polarity of PFN can be adjusted by replacing the neutral amines with ionic species. A cationic-type derivative, $\mathrm{PFNBr}$, and an anionictype derivative, PFCOONa, are synthesized to produce opposite polarities. ${ }^{32,33}$ By scanning the surface WF on PFNBr/Al and PFCOONa/Al, opposite dipoles are observed. The electric potential shift caused by the dipole at the PFNBr/Al interface is found to be $\sim 0.23 \mathrm{eV}$. The dipole direction is orientated to reduce the Al WF. At the PFCOONa/Al interface, the WF change is $\sim-0.04 \mathrm{eV}$, showing that the dipole direction is orientated to weakly increase the Al WF (XPS spectrum in Supplementary S11a). The measured dipoles 

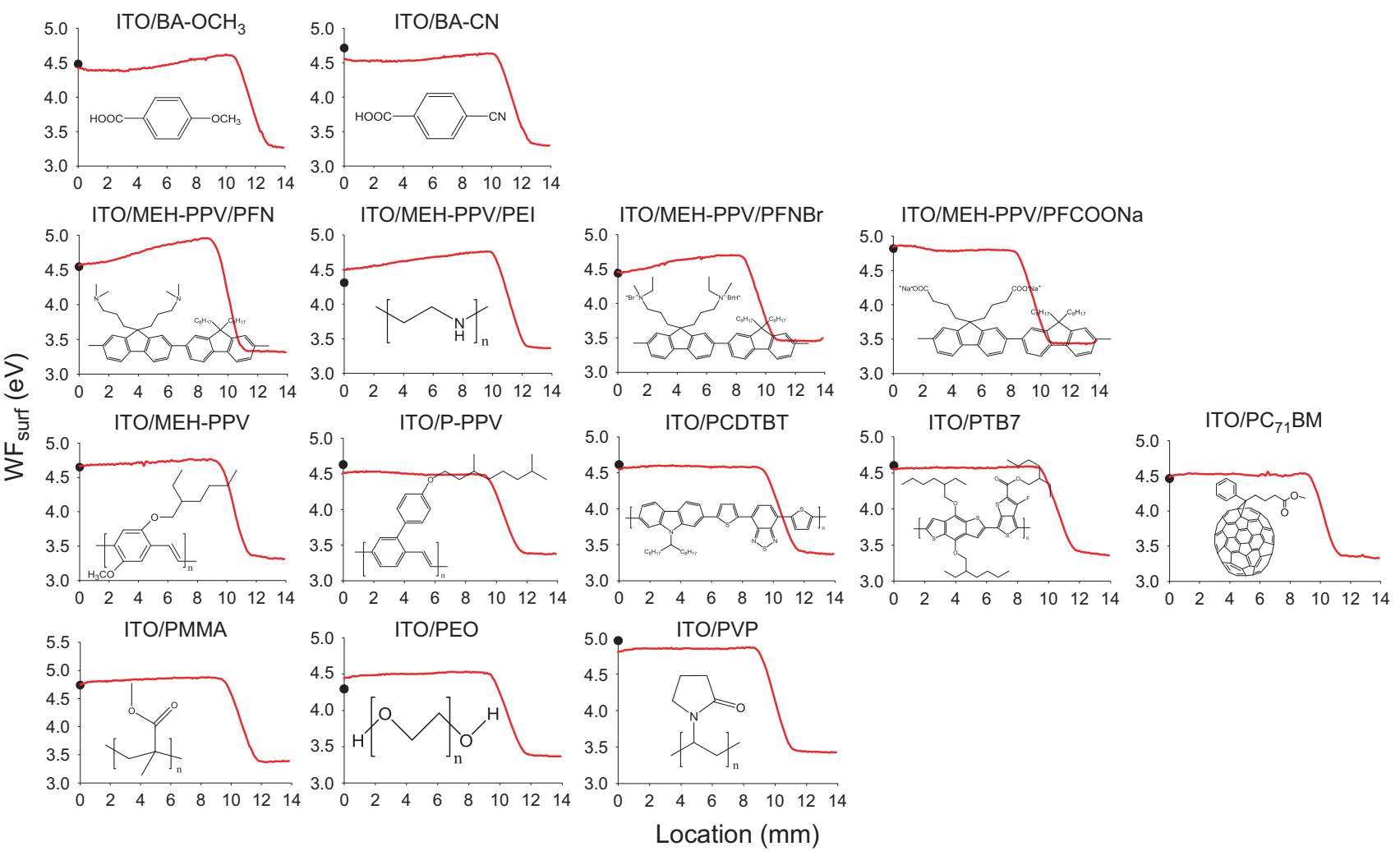

Figure 5 Surface work function (WF) scan plots (red line) from side to side on the organic/post-deposited Al samples. The organic layers are typical materials with conjugated and nonconjugated, polar and nonpolar and semiconducting and insulating properties. The black dots in the plots indicate the surface WF of the organic layer before metal deposition. A typical variation of $\pm 0.1 \mathrm{eV}$ should be considered because of variations in sample preparation and measurement errors.

confirm their use as an electron injection material and hole injection material, respectively. ${ }^{33,34}$

Active materials in organic electronic devices are usually less polar. Popular light-emitting materials, such as MEH-PPV and P-PPV, and popular light-harvesting materials, such as PCDTBT, PTB7 and $\mathrm{PC}_{71} \mathrm{BM}$, are examined. As shown in Figure 5, a small difference of $<0.1 \mathrm{eV}$ is observed in the surface WFs of the less polar active materials before and after Al deposition. For insulating polymers, such as PMMA, no WF modification effect is observed. However, at the $\mathrm{PEO} / \mathrm{Al}$ interface, a dipole directed from the metal to the organic layer is observed, confirming the coordination interaction between $\mathrm{Al}$ and the ethylene oxide group, indicating that PEO is a good cathode interfacial material. ${ }^{35,36}$ For PVP, an opposite dipole that increases the $\mathrm{Al}$ WF is found, confirmed by a slight shift in the $\mathrm{Al} 2 \mathrm{p}$ peak to higher binding energy (Supplementary Figure 11b). The dipole may have arisen from a weak coordinative interaction between the $\mathrm{C}-\mathrm{N}$ group of PVP and the metal and/or hydrogen bonding from $\mathrm{C}=\mathrm{O}$ to the oxidized surface of the metal. ${ }^{37,38}$ The surface WF changes of various materials show that the presence of polar species is key to producing a dipole at the organic/metal interface. Moreover, the dipole direction can be adjusted by changing the polar species.

The surface WF of organic materials spin-coated on pre-deposited $\mathrm{Al}$ substrates are measured to examine the dipole at organic/ pre-deposited metal interfaces. Shown in Figure 6, potential changes at interfaces with pre-deposited and post-deposited $\mathrm{Al}$ are compared. A positive value indicates that the dipole is directed from the metal to the organic layer, whereas a negative value implies that the dipole is directed from the organic layer to the metal. For most organic materials, the dipoles at both interfaces are very similar, and this is expected because of the similar surface chemistry of $\mathrm{Al}$ at both interfaces, where the Al surface is spontaneously oxidized. ${ }^{39}$ Some organic materials, such as PTB7 and PEO, exhibit small differences in the surface WF that can be attributed to the different contact morphologies at the organic/metal interface. For pre-deposited $\mathrm{Al}$, the interface is distinct without intermixing of organic and metal molecules. For post-deposited $\mathrm{Al}$, hot $\mathrm{Al}$ atoms may diffuse into the organic layer disturbing the molecular stacking and forming C-Al bonds. ${ }^{16,18,19}$

As shown in Figure 6, the dipole at the $\mathrm{PC}_{71} \mathrm{BM} / \mathrm{Al}$ interface is markedly different between the pre-deposited $\mathrm{Al}$ and post-deposited $\mathrm{Al}$ samples. Spin-coated $\mathrm{PC}_{71} \mathrm{BM}$ on pre-deposited $\mathrm{Al}$ gives a surface WF of $4.2 \mathrm{eV}$, increasing the potential of the bare $\mathrm{Al}$ surface by $\sim 0.8 \mathrm{eV}$. In contrast, the WF does not change when depositing an ultrathin $\mathrm{Al}$ layer on $\mathrm{PC}_{71} \mathrm{BM}$. The surface WF change at the $\mathrm{PC}_{71} \mathrm{BM}$ /pre-deposited $\mathrm{Al}$ interface is attributed to Fermi-level pinning. ${ }^{21,39}$ As the surface WF of oxidized $\mathrm{Al}$ is $\sim 3.4 \mathrm{eV}$, which is smaller than the $\mathrm{E}_{\mathrm{ICT}}$ - level of $\mathrm{PC}_{71} \mathrm{BM}$, the Fermi level of $\mathrm{Al}$ is hence pinned to the $\mathrm{E}_{\mathrm{ICT}}$ - level at $-4.2 \mathrm{eV}$ by forming a dipole directed from the organic layer to the metal, as shown in Figure 1c. At the $\mathrm{PC}_{71} \mathrm{BM} /$ post-deposited $\mathrm{Al}$ interface, the interaction between $\mathrm{PC}_{71} \mathrm{BM}$ and the $\mathrm{Al}$ species produces a negligible dipole. A detailed discussion about the interface is provided in the Supplementary Information in Supplementary Figures S12 and S13. The different results for the pre-deposited and post-deposited Al interfaces show that this method for measuring the potential change in ultrathin metals is able to distinguish two dipole formation mechanisms: one is 


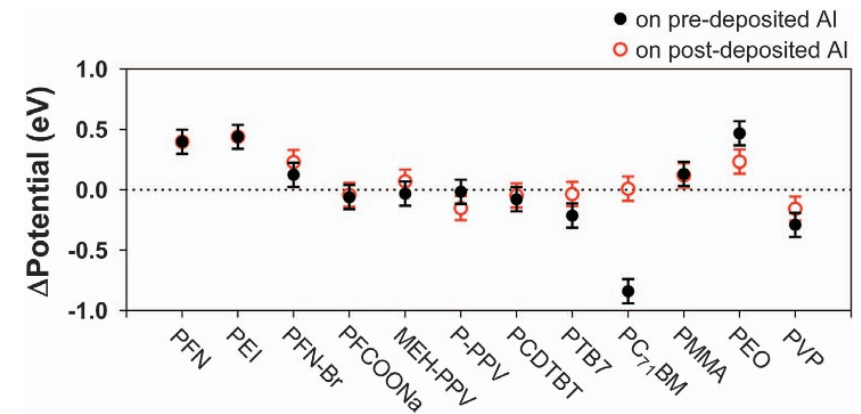

Figure 6 Surface potential changes at the organic/post-deposited Al interface and the organic/pre-deposited Al interface are compared. Positive values correspond to a dipole direction from the metal to the organic layer, and vice versa. The error bar is $\pm 0.1 \mathrm{eV}$.

through molecule-molecule interactions, whereas the other is through Fermi-level pinning.

\section{CONCLUSION}

In summary, a novel method to probe dipole formation at the organic/ post-deposited metal interface is demonstrated. In the method, an ultrathin Al film is obtained by metal evaporation through a slit opening between the sample substrate and the shadow mask. As the ultrathin $\mathrm{Al}$ layer does not screen out the dipole, measurement of the surface WF across the sample surface reveals dipole formation at the organic/post-deposited metal interface that is then compared with the dipole formed at the organic/pre-deposited metal interface. Dipole formation can arise from the interaction of the organic polar species with the metal surface, Fermi-level pinning-induced electron transfer or the interface morphology. It is found that for most organic materials, the dipoles at the two types of interfaces are very similar. At the $\mathrm{PC}_{71} \mathrm{BM} / \mathrm{Al}$ interface, however, there is no significant dipole detected on the post-deposited $\mathrm{Al}$, attributed to the complex interface between $\mathrm{PC}_{71} \mathrm{BM}$ and $\mathrm{Al} / \mathrm{Al}_{2} \mathrm{O}_{3}$ molecules. The large dipole at the $\mathrm{PC}_{71} \mathrm{BM} /$ pre-deposited $\mathrm{Al}$ interface originates from the Fermi-level pinning of metallic $\mathrm{Al}$ to the $\mathrm{PC}_{71} \mathrm{BM} \mathrm{E}_{\mathrm{ICT}}$ - level. The method proposed in this contribution enables the quick and easy assessment of the dipole formed at the organic/metal interface that guides the selection of materials for application in organic electronics devices.

\section{CONFLICT OF INTEREST}

The authors declare no conflict of interest.

\section{ACKNOWLEDGEMENTS}

We are deeply grateful to the National Key Basis Research and Development Program of China (973 program, Grant No. 2015CB655004) founded by MOST, the National Key Research and Development Program of China (2016YFB0401400), the National Natural Science Foundation of China (51573056, 51373057) and the Science and Technology Project of Guangdong Province (2015B090915001) for their financial support.

1 Ishii, H., Sugiyama, K., Ito, E. \& Seki, K. Energy level alignment and interfacial electronic structures at organic/metal and organic/organic interfaces. Adv. Mater. 11, 605-625 (1999).

2 Thompson, B. C. \& Fréchet, J. M. J. Polymer-fullerene composite solar cells. Angew. Chemie Int. Ed. 47, 58-77 (2008).

3 Cao, Y., Yu, G., Parker, I. D. \& Heeger, A. J. Ultrathin layer alkaline earth metals as stable electron-injecting electrodes for polymer light emitting diodes. J. Appl. Phys. 88, 3618-3623 (2000).

4 Parker, I. D. Carrier tunneling and device characteristics in polymer lightemitting diodes. J. Appl. Phys. 75, 1656-1666 (1994).
5 Brabec, C. J., Cravino, A., Meissner, D., Sariciftci, N. S., Fromherz, T., Rispens, M. T., Sanchez, L. \& Hummelen, J. C. Origin of the open circuit voltage of plastic solar cells. Adv. Funct. Mater. 11, 374-380 (2001).

6 Yip, H.-L. \& Jen, A. K.-Y. Recent advances in solution-processed interfacial materials for efficient and stable polymer solar cells. Energy Environ. Sci. 5, 5994-6011 (2012).

7 Huang, F., Wu, H. \& Cao, Y. Water/alcohol soluble conjugated polymers as highly efficient electron transporting/injection layer in optoelectronic devices. Chem. Soc. Rev. 39, 2500-2521 (2010).

8 Zhou, Y., Fuentes-Hernandez, C., Shim, J., Meyer, J., Giordano, A. J., Li, H., Winget, P., Papadopoulos, T., Cheun, H., Kim, J., Fenoll, M., Dindar, A., Haske, W., Najafabadi, E., Khan, T. M., Sojoudi, H., Barlow, S., Graham, S., Brédas, J.-L., Marder, S. R., Kahn, A. \& Kippelen, B. A universal method to produce low-work function electrodes for organic electronics. Science 336, 327-332 (2012).

9 Sun, C., Wu, Z., Yip, H.-L., Zhang, H., Jiang, X.-F., Xue, Q., Hu, Z., Hu, Z., Shen, Y., Wang, M., Huang, F. \& Cao, Y. Amino-functionalized conjugated polymer as an efficient electron transport layer for high-performance planar-heterojunction perovskite solar cells. Adv. Energy Mater. 6, 1501534 (2015).

10 Wang, Q., Zhou, Y., Zheng, H., Shi, J., Li, C., Su, C. Q., Wang, L., Luo, C., Hu, D., Pei, J., Wang, J., Peng, J. \& Cao, Y. Modifying organic/metal interface via solvent treatment to improve electron injection in organic light emitting diodes. Org. Electron. 12, 1858-1863 (2011).

11 Chen, Y., Hu, Z., Zhong, Z., Shi, W., Peng, J., Wang, J. \& Cao, Y. Aqueous solution processed, ultrathin ZnO Film with low conversion temperature as the electron transport layer in the inverted polymer solar cells. J. Phys. Chem. C 118, 21819-21825 (2014).

12 Jiang, Z., Zhong, Z., Xue, S., Zhou, Y., Meng, Y., Hu, Z., Ai, N., Wang, J., Wang, L., Peng, J., Ma, Y., Pei, J., Wang, J. \& Cao, Y. Highly efficient, solution processed electrofluorescent small molecule white organic light-emitting diodes with a hybrid electron injection layer. ACS Appl. Mater. Interfaces 6, 8345-8352 (2014).

13 Lee, B. H., Jung, I. H., Woo, H. Y., Shim, H.-K., Kim, G. \& Lee, K. Multi-charged conjugated polyelectrolytes as a versatile work function modifier for organic electronic devices. Adv. Funct. Mater. 24, 1100-1108 (2013).

14 Liu, F., Page, Z. A., Duzhko, V. V., Russell, T. P. \& Emrick, T. Conjugated polymeric zwitterions as efficient interlayers in organic solar cells. Adv. Mater. 25, 6868-6873 (2013).

15 Wang, H., Amsalem, P., Heimel, G., Salzmann, I., Koch, N. \& Oehzelt, M. Bandbending in organic semiconductors: the role of alkali-halide interlayers. Adv. Mater. 26, 925-930 (2013).

16 Salaneck, W. R. \& Brédas, J.-L. The metal-on-polymer interface in polymer light emitting diodes. Adv. Mater. 8, 48-52 (1996).

17 Kampen, T. U., Das, A., Park, S., Hoyer, W. \& Zahn, D. R. T. Relation between morphology and work function of metals deposited on organic substrates. Appl. Surf. Sci. 234, 333-340 (2004).

18 Paez, B. A., Salvan, G., Silaghi, S., Scholz, R., Kampen, T. U. \& Zahn, D. R. T. Raman monitoring of In and Ag growth on PTCDA and DiMe-PTCDI thin films. Appl. Surf. Sci. 234, 168-172 (2004).

19 Hirose, Y., Kahn, A., Aristov, V. \& Soukiassian, P. Chemistry, diffusion, and electronic properties of a metal/organic semiconductor contact: In/perylenetetracarboxylic dianhydride. Appl. Phys. Lett. 68, 217-219 (1996).

20 Osikowicz, W., de Jong, M. P., Braun, S., Tengstedt, C., Fahlman, M. \& Salaneck, W. R. Energetics at Au top and bottom contacts on conjugated polymers. Appl. Phys. Lett. 88, 193503-193504 (2006).

$21 \mathrm{Hu}$, Z., Zhong, Z., Chen, Y., Sun, C., Huang, F., Peng, J., Wang, J. \& Cao, Y. Energylevel alignment at the organic/electrode interface in organic optoelectronic devices. Adv. Funct. Mater. 26, 129-136 (2016).

22 Vancea, J., Reiss, G., Butz, D. \& Hoffmann, H. Thickness-dependent effects in the work function of polycrystalline Cu-films. Europhys. Lett. 9, 379-384 (1989).

23 Lazzaroni, R., Brédas, J. L., Dannetun, P., Lögdlund, M., Uvdal, K. \& Salaneck, W. R. Electronic structure of the aluminum/polythiophene interface: A joint experimental and theoretical study. Synth. Met. 43, 3323-3328 (1991).

24 Hirose, Y., Kahn, A., Aristov, V., Soukiassian, P., Bulovic, V. \& Forrest, S. R. Chemistry and electronic properties of metal-organic semiconductor interfaces: Al, Ti, In, Sn, Ag, and Au on PTCDA. Phys. Rev. B 54, 13748-13758 (1996).

25 Liu, C., Wang, K., Gong, X. \& Heeger, A. J. Low bandgap semiconducting polymers for polymeric photovoltaics. Chem. Soc. Rev. 45, 4825-4846 (2015).

26 Braun, S., Salaneck, W. R. \& Fahlman, M. Energy-level alignment at organic/metal and organic/organic interfaces. Adv. Mater. 21, 1450-1472 (2009).

27 Frey, H. \& Khan, H. R. Handbook of Thin Film Technology, (Springer, Berlin/ Heidelberg, Germany, 2010).

28 Carrara, M., Nüesch, F. \& Zuppiroli, L. Carboxylic acid anchoring groups for the construction of self-assembled monolayers on organic device electrodes. Synth. Met. 121, 1633-1634 (2001).

29 Yip, H.-L., Hau, S. K., Baek, N. S., Ma, H. \& Jen, A. K.-Y. Polymer solar cells that use self-assembled-monolayer-modified $\mathrm{ZnO} /$ metals as cathodes. Adv. Mater. 20, 2376-2382 (2008).

30 Vilan, A. \& Cahen, D. Soft contact deposition onto molecularly modified GaAs. Thin metal film flotation: principles and electrical effects. Adv. Funct. Mater. 12, 795-807 (2002).

31 Zhong, Z., Hu, Z., Jiang, Z., Wang, J., Chen, Y., Song, C., Han, S., Huang, F., Peng, J., Wang, J. \& Cao, Y. Hole-trapping effect of the aliphatic-amine based electron injection materials in the operation of OLEDs to facilitate the electron injection. Adv. Electron. Mater. 1, 1400014 (2015). 
32 Duan, C., Zhang, K., Zhong, C., Huang, F. \& Cao, Y. Recent advances in water/alcoholsoluble $\pi$-conjugated materials: new materials and growing applications in solar cells. Chem. Soc. Rev. 42, 9071-9104 (2013).

33 Zhang, K., Hu, Z., Xu, R., Jiang, X.-F., Yip, H.-L., Huang, F. \& Cao, Y. High-performance polymer solar cells with electrostatic layer-by-layer self-assembled conjugated polyelectrolytes as the cathode interlayer. Adv. Mater. 27, 3607-3613 (2015).

34 Kang, R., Oh, S.-H. \& Kim, D.-Y. Influence of the ionic functionalities of polyfluorene derivatives as a cathode interfacial layer on inverted polymer solar cells. ACS Appl. Mater. Interfaces 6, 6227-6236 (2014).

35 Lee, T.-H., Huang, J.-C.-A., Pakhomov, G. L., Guo, T.-F., Wen, T.-C., Huang, Y.-S., Tsou, C.-C., Chung, C.-T., Lin, Y.-C. \& Hsu, Y.-J. Organic-oxide cathode buffer layer in fabricating high-performance polymer light-emitting diodes. Adv. Funct. Mater. 18, 3036-3042 (2008).

36 Song, Q., Song, C., Zhong, Z., Hu, Z., Wang, L., Wang, J. \& Cao, Y. Ether solvent treatment to improve the device performance of the organic light emitting diodes with aluminum cathode. Org. Electron. 24, 241-245 (2015).

37 Gottapu, S., Padhi, S. K., Krishna, M. G. \& Muralidharan, K. Poly(vinylpyrrolidone) stabilized aluminum nanoparticles obtained by the reaction of $\mathrm{SiCl} 4$ with $\mathrm{LiAlH} 4$. New J. Chem. 39, 5203-5207 (2015).
38 Jing, C. \& Hou, J. Sol-gel-derived alumina/polyvinylpyrrolidone hybrid nanocomposite film on metal for corrosion resistance. J. Appl. Polym. Sci. 105, 697-705 (2007).

39 Osikowicz, W., de Jong, M. P. \& Salaneck, W. R. Formation of the interfacial dipole at organic-organic interfaces: C60/polymer interfaces. Adv. Mater. 19, 4213-4217 (2007).

(c) (i) This work is licensed under a Creative Commons Attribution 4.0 International License. The images or other third party material in this article are included in the article's Creative Commons license, unless indicated otherwise in the credit line; if the material is not included under the Creative Commons license, users will need to obtain permission from the license holder to reproduce the material. To view a copy of this license, visit http:// creativecommons.org/licenses/by/4.0/

(C) The Author(s) 2017

Supplementary Information accompanies the paper on the NPG Asia Materials website (http://www.nature.com/am) 